\title{
Chapter 19 \\ Impressions of the Forestry Managers and Students Who Participated in the Radioactivity Damage Investigation of Forests
}

\author{
Eihachi Horiuchi, Kiyoaki Sasaki, Masaaki Itakura, Chisato Yasukawa, \\ and Chihiro Kinoshita
}

\begin{abstract}
This chapter portrays the distress of owners and workers of radiationcontaminated forests and the sense of fear and mission felt by students who participated in forest field surveys in such severe environments.
\end{abstract}

Keywords Radioactive contamination in forests • Forest owners • Forestry cooperatives $\bullet$ Measuring radioactive content of trees

\subsection{The Ordeal Inflicted by the Great East Japan Earthquake and the Spread of Radiation}

Eihachi Horiuchi

Soma Regional Forestry Cooperative, Nishiki-cho, Haramati-ku, Minamisoma, Fukushima 975-0031, Japan

Soma's regional forestry cooperative is located in the north of Fukushima's Hamadori region and is responsible for an area spanning two cities and one town. Our cooperative, which has a membership of around 2,600, works to

\footnotetext{
E. Horiuchi $(\bowtie)$

Soma Regional Forestry Cooperative, Nishiki-cho, Haramati-ku, Minamisoma, Fukushima 975-0031, Japan

K. Sasaki

Temporary house, Ushigoe, Haramati-ku, Minamisoma, Fukushima 975-0017, Japan

M. Itakura $\bullet$ C. Yasukawa $\bullet$ C. Kinoshita

Department of Bioscience, Tokyo University of Agriculture,

1-1-1 Sakuragaoka, Setagaya-ku, Tokyo 156-8502, Japan

(C) The Author(s) 2015

T. Monma et al. (eds.), Agricultural and Forestry Reconstruction

After the Great East Japan Earthquake, DOI 10.1007/978-4-431-55558-2_19
} 
conserve forests and increase production output in the approximately 34,000 ha of forest (approximately 22,000 ha of which is privately owned) in the area. The Great East Japan Earthquake of March 11, 2011 led to explosions at the Tokyo Electric Power Company (TEPCO) nuclear power station, which is located to our south. Because of the myth of safety surrounding nuclear power, nobody in their worst nightmares imagined that radiation would spread or that people would be forced to evacuate, a mindset that led to secondary damage. Two to three days after the accident, all Minamisoma's residents were ordered to evacuate, including those living outside the $30-\mathrm{km}$ zone. All facilities serving the public were closed, with the exception of government organizations such as municipal offices, and major panic ensued as distribution of products came to a halt. When the spread of radioactive material began to ease from around March 20 , we began recovery work such as searching for missing persons outside the 30-km zone and using forestry machinery to clear debris (we also requested assistance from other cooperatives.)

At the beginning of July, when the situation was looking dire, Professor Takahisa Hayashi of Tokyo University of Agriculture visited our office and offered to conduct a study of radioactive contamination from the nuclear accident in forests and trees. We gratefully accepted. As a result we learned that the soil, litter layer, and trees were contaminated with high concentrations of radioactive cesium, and we appealed to Fukushima's prefectural forestry association and other cooperatives, pointing out that the state of radiation contamination in forests should be studied and ascertained. We held briefings on damage compensation at each cooperative under the prefectural association, and are currently in the process of filing for compensation. We also appealed to other organizations such as the Forestry Agency and the Forestry and Forest Products Research Institute, pointing out that the presence of contamination hot spots in mountain forests meant that ongoing and detailed studies were needed. In March of the following year the Forestry Agency and the Forestry and Forest Products Research Institute finally conducted studies at 12 sites in Fukushima Prefecture, and in August the Forestry Agency released the study results-just once. The highest concentration of cesium was $497 \mathrm{~Bq} / \mathrm{kg}$, measured in Japanese red pine sapwood in the Ohara district of Haramachi in Minamisoma. As a high ambient radiation dose in the forest corresponds to a high cesium concentration in the bark and wood, we also requested investigations at a further two sites. However, this request was turned down. 


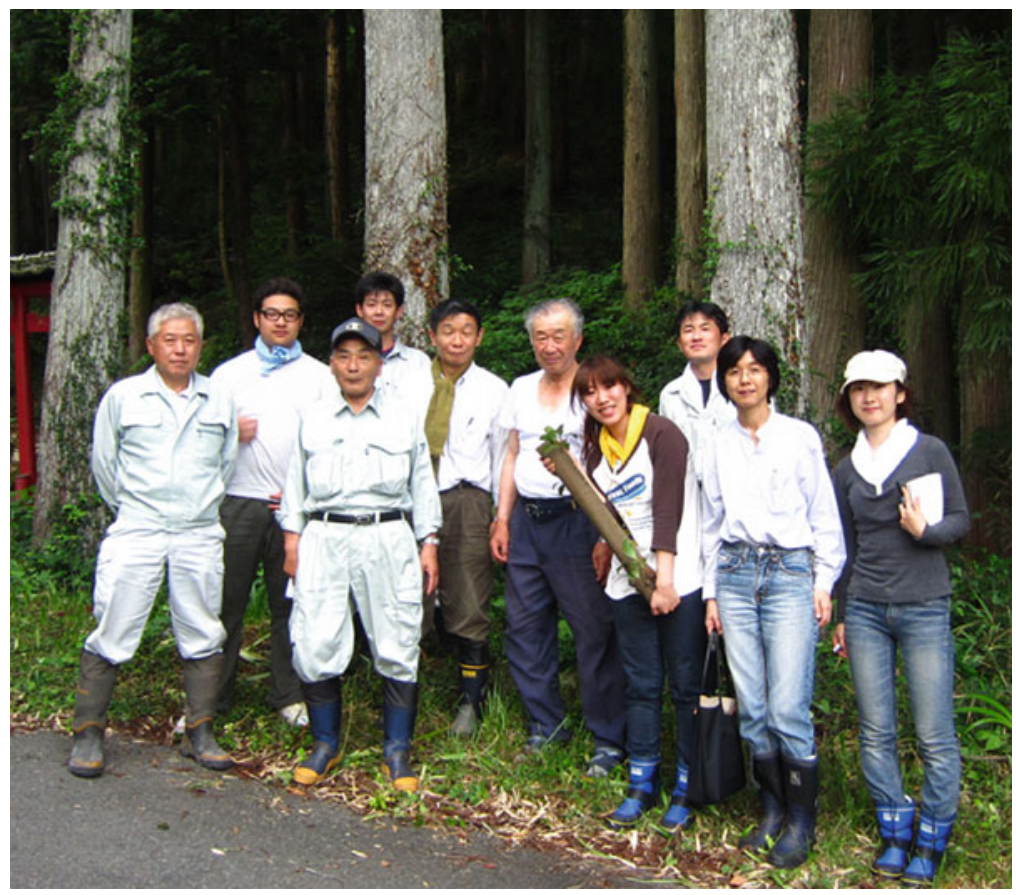

About 10 months later, when Professor Hayashi retested Japanese cedar, Japanese red pine, and other trees in forests in the same Ohara district in which the Forestry Agency study was conducted, some trees were found to have around three times the cesium previously reported. Subsequently, Professor Hayashi leased an area of forest in the Ogai district of Haramachi, Minamisoma, where he has planted a variety of saplings to study the absorption of radioactive material during their growth stages. He is also conducting ongoing trials that involve sprinkling water-dispersible potassium chloride and ammonium nitrate powder on trees via radio-controlled helicopter and monitoring their radiation absorption.

However, there has been no progress on compensation for the damage to our forests because TEPCO has not set compensation standards for forest-related damage. Our young successors are despondent. Despite having inherited these assets from their forebears, the radiation-contaminated wood cannot be used, and their forests have lost their value. Looking ahead, I am worried about how to develop plans for rehabilitating, consolidating, and operating our forests as a business, and whether we will be able to obtain the understanding and cooperation of forest owners. 


\subsection{An Oasis in the Desert}

Kiyoaki Sasaki

Temporary house, Ushigoe, Haramati-ku, Minamisoma, Fukushima 975-0017, Japan

I previously lived in a small village $16 \mathrm{~km}$ from the nuclear power station. It has now been designated a long-term evacuation zone, and the region around the village has been designated a potentially permanent evacuation zone. I own a forest of approximately 40 ha, which my family has worked hard to raise and beautify over generations, in between farming. We planted pine trees on the flat land, and although that pine forest was our pride and joy, it suffered damage from pests. After a range of measures, including changing tree varieties, our trees finally reached the pruning stage. The woods surrounding our house cover approximately 1 ha, and are now made up of giant trees of the Japanese cedar, Japanese zelkova, and momi fir varieties.

However, this fruit of my ancestors' labor was instantly made worthless by the explosions at the nuclear plant. Although crop and livestock farmers suffered similar damage, they have strong backing from agricultural and livestock industry groups, local governments, and government bodies, and I hear that they have managed to win compensation. However, forestry was the only sector in which industry groups and related organizations stayed silent, and we could not even get a proper investigation into the extent of contamination. The days I spent in Fukushima City after being evacuated were filled with despair.

In January 2012, nearly a year after the nuclear accident, a legal advisor for the Fukushima prefectural forestry association held a briefing session on compensation issues. At the same meeting, Professor Takahisa Hayashi from Tokyo University of Agriculture explained the results of studies into the extent of contamination. I did not know anything about the science involved, so I asked him straight out whether our trees could no longer be sold as products. Professor Hayashi's reply was "That's probably the way it's going to end up." I got the impression that the legal advisor's explanation was not in line with the actual damage that had occurred.

Naturally, there was no proactive discussion from the association, and it seemed as though they were deliberately being vague about the real state of the damage. I traveled to the association's headquarters in Fukushima City together with Takeyama, another forestry operator, and we asked them to reconsider their policy. They replied that they had recently made an approach to TEPCO regarding compensation, but there have been no subsequent developments, and I have doubts about how serious they really are. 


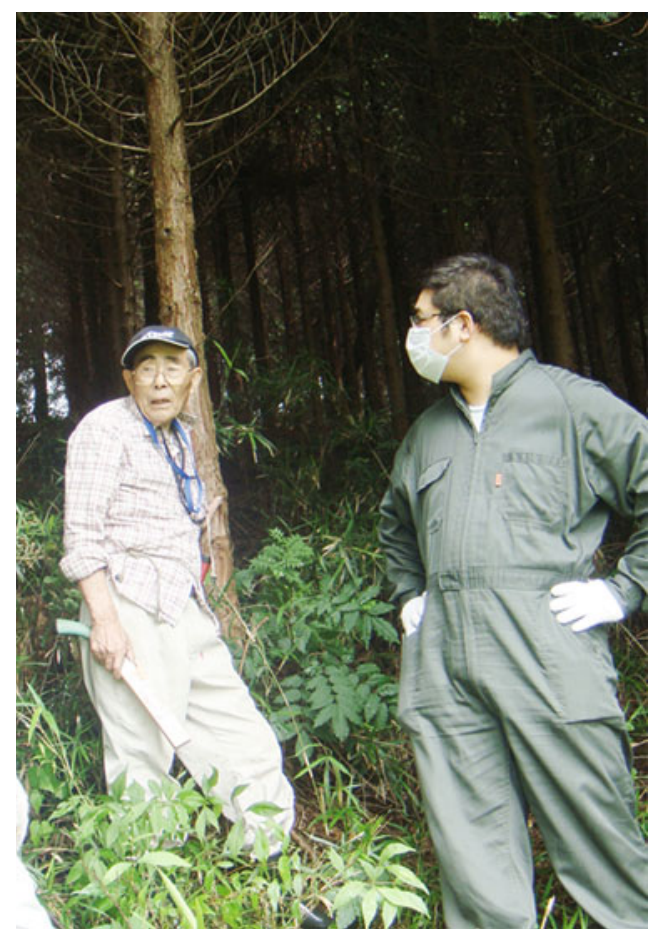

Given these circumstances, we have been gathering volunteers since last year and have decided to take action. If the worst comes to the worst, we are considering filing an independent lawsuit. But it is impossible for untrained people to measure the contamination levels. Thankfully, Professor Hayashi informed us that it is Tokyo University of Agriculture's policy to proactively study contamination levels free of charge. As a result, we have benefited from highly dedicated investigation of those levels right through to the present. It has been like finding an oasis in the desert. If it develops into a court case and we win compensation, I hope to donate to Professor Hayashi's research group.

\title{
19.3 The Difficulty of Assessing Radioactive Contamination in Forests
}

\author{
Masaaki Itakura \\ Department of Bioscience, Tokyo University of Agriculture, \\ 1-1-1 Sakuragaoka, Setagaya-ku, Tokyo 156-8502, Japan
}

From our base in Minamisoma, we conducted studies of forests in the town of Shinchi and the cities of Soma and Minamisoma. In contrast to its support for regular farmers, the government's support for local forestry operators was given a low 
priority, and operators were unable to ascertain the degree of contamination to the trees they had often spent 50 years or more raising, data that are required to receive compensation. We therefore provided local forestry operators with information on this invisible radioactive contamination in the form of accurate values and tried to help them obtain the appropriate compensation. To do this, it was essential that we accurately identify the extent to which the trees were contaminated.

One fact that became clear from studying trees in various districts was that forest contamination was not proportional to the ambient dose. In some areas we found highly concentrated contamination in the wood despite a low air reading, and vice versa. However, at present the ambient dose is used to determine forest contamination, and in some cases forestry operators are not able to receive compensation for their trees despite them being too contaminated to ship to market. We thought, therefore, that to accurately identify the radiation level of all of Fukushima Prefecture's forests, we needed to take dosage measurements of trees and soil in subdivided plots. This realization brought home the difficulty of identifying contamination levels in vast forest areas. As the field of study was radiation, which was not our area of specialization, the field work was initially one ordeal after another.

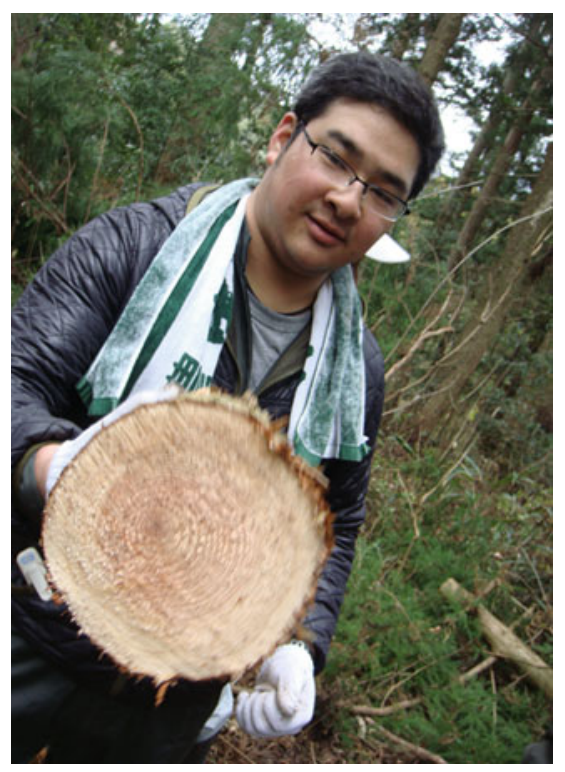

The first hurdle was the fact that, in contrast to the crop fields, we faced a harsh working environment, which included steep slopes, plants that could cause skin irritation—such as Japanese sumac trees-or thorns, and insects such as hornets and horseflies. One of our students was unable to walk for 3 days after being stung by an insect. Experiencing this work environment firsthand quickly made me realize that decontaminating the area using human labor was not realistic. I felt a strong need to consider biological methods of decontaminating the area. 
Another surprise was the strength of the radiation dose. In some forest areas it was more than ten times higher than in urban areas. Working in these areas brought constant concern about radiation exposure. To dispel this worry I remember telling myself that a return flight from Tokyo to New York would expose me to about $200 \mu \mathrm{Sv}$ of radiation but working in the forest for 3 days would expose me to only $30 \mu \mathrm{Sv}$ at most, so it was no great concern. It was a shock when my girlfriend at the time asked me not to go to Fukushima.

But I lied, telling her I was going to the university's research forest before heading to Fukushima. I do not regret doing this. As a matter of fact, when our personal internal radiation exposure was measured using a full-body counter, none of the students, including myself, returned abnormal readings.

Consequently, while I was still a student, I was able to experience a crisis situation such as this up close, take action, and learn the importance of facing up to difficulties. Forests act as a dam for radioactive material and I believe that assessing their contamination will be essential for the restoration effort in Fukushima to go forward.

\title{
19.4 Studying Forest Radiation Contamination Changed My Outlook on Life
}

\author{
Chisato Yasukawa \\ Department of Bioscience, Tokyo University of Agriculture, \\ 1-1-1 Sakuragaoka, Setagaya-ku, Tokyo 156-8502, Japan
}

In July 2011, as a third-year student, I began researching forest restoration, working in the forests of Minamisoma, Fukushima Prefecture. Although our task was forest restoration, in reality this initially involved confirming the level of contamination in each area. We trudged around the nearby woodland, survey meters in hand. There were large areas of contamination, even in the peaceful, refreshing woodland, and I felt the invisible fear of radiation. I was motivated by a desire to see the situation with my own eyes, and to do something to help. I felt the pain of families split apart and people losing their homes, and the distress of seeing the trees one had lovingly raised over many years contaminated with radiation.

I studied ways to decontaminate affected forests as quickly as possible. Our experiments confirmed that feeding nitrogen to plants in a nutrient-poor medium such as forest soil caused them to absorb a large amount of cesium. After conducting forest experiments with a range of tree varieties, we hypothesized a model in which planting certain tree types would help complete the decontamination process more quickly than simply leaving the forest as it was. Meanwhile, my autoradiography experiments to observe cesium in the vascular bundles of plants ended in failure time after time, and I felt like crying from frustration. Although the professors are demanding when it comes to experiments, I was able to keep going thanks to the 
support of my peers and the more senior students. I also gave slide-based presentations at the annual meeting of the Japanese Society of Plant Physiologists when I was a third-year student, and at the annual meeting of the Japan Wood Research Society when I was in my fourth year. While I gained much from performing practical-science-based research, therefore, I also realized how academia and science are closely linked to society and the world at large.

A couple of years on from the nuclear accident, people opposed to the country's nuclear reactors being restarted hold protests in Tokyo on an almost weekly basis. I have begun attending these demonstrations. The ideals of the participants connected directly with me, and I have become strongly opposed to nuclear power. To be honest, it took the nuclear accident for me to take an interest. The event awakened me to a new way of life. Although it is possible to live life without being aware of important issues, as members of society I believe we should consider issues from our own perspective, make choices, and be prepared to speak up if necessary.

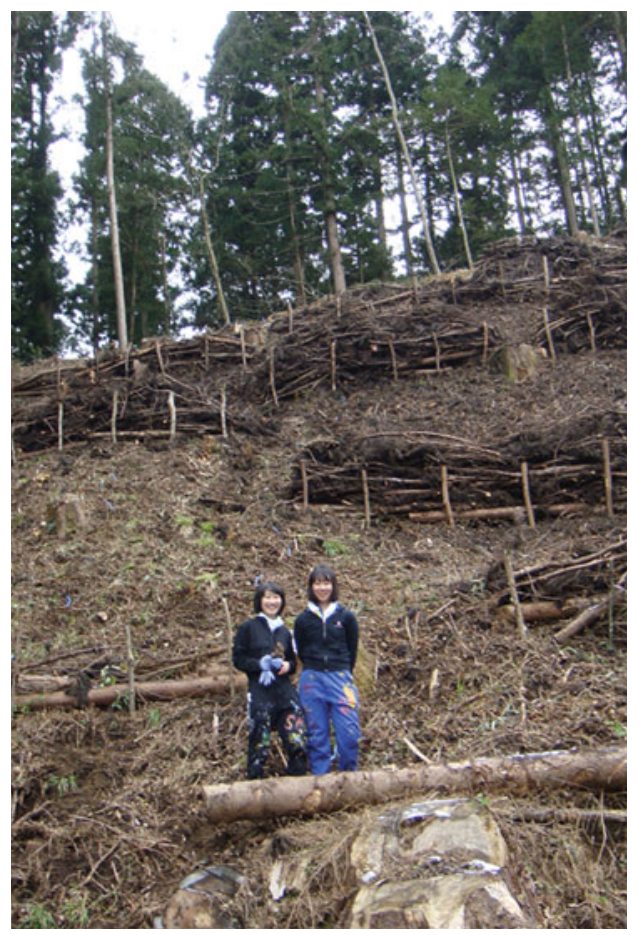

My research led me to think about Fukushima, about radioactive cesium and our forests, and about society. As a member of the generation responsible for tackling Japan's radiation problems, I hope to put this experience to good use in future. 


\title{
19.5 Finding a Way to Live with Cesium
}

\author{
Chihiro Kinoshita \\ Department of Bioscience, Tokyo University of Agriculture, \\ 1-1-1 Sakuragaoka, Setagaya-ku, Tokyo 156-8502, Japan
}

I was in my first year of university when the Great East Japan Earthquake struck. The tsunami that followed the earthquake was a terrible tragedy in itself, and then the radioactive contamination from the nuclear disaster was even more of a concern. I spent days in anguish about what to do-I considered volunteering, then found myself questioning what I could possibly offer in the way of assistance, and wondering whether I was just seeking my own self-satisfaction. The problem was-and this is perhaps not the best way to put it—my image of volunteer work consisted of groups pulling up in buses just to do one-off, simple work, feel pleased with themselves, and pretend to be chummy with each other.

But after being assigned to the Laboratory of Plant Molecular Genetics, I learned about the initiatives the laboratory was undertaking in Fukushima and plunged straight into the action. What awaited me was a world of practical science far removed from the "pretend" one-off volunteer work I had imagined. Our research aimed to prevent the transfer of radioactive cesium to fruit in the city of Date in Fukushima Prefecture, and communication with the professors and the more experienced students at the work site was as real as it could be. We performed experiments jointly with representatives from JA Datemirai (a local agricultural cooperative) and companies. As we worked in the fields, members of the public passing by would stop and talk to us. At first I could not understand what they were saying because I did not know the Tohoku dialect, but as they continued to stop and offer us encouragement, I gradually got used to the local accent.

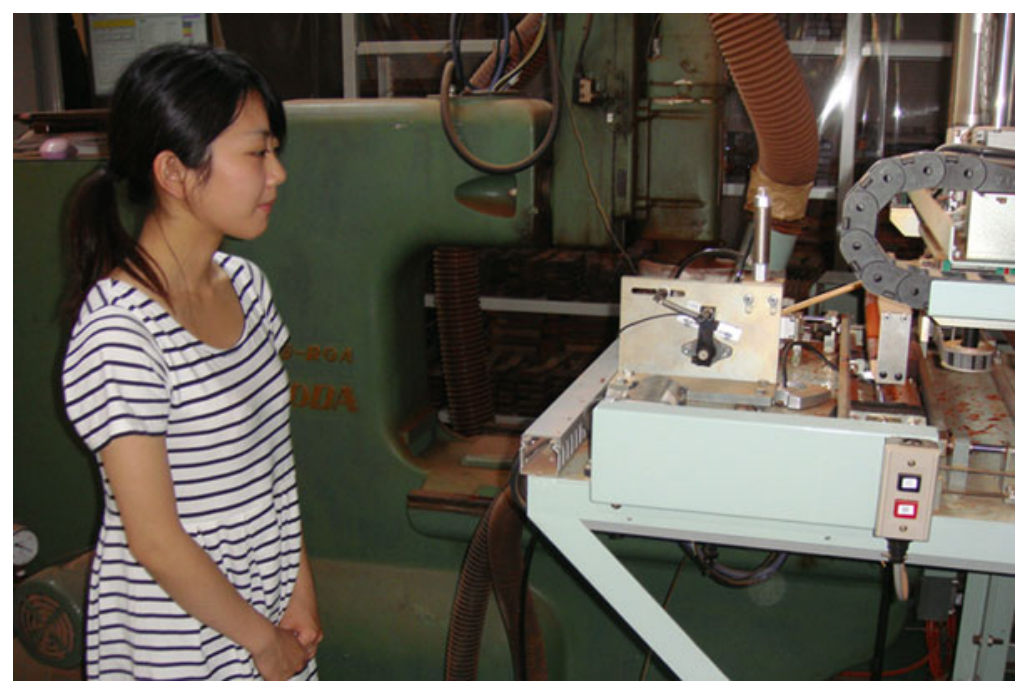


I believe that the research we are performing in the field is really important. We are attempting techniques that, if they prove successful, can be applied on a global level in a range of countries and regions. Our efforts involve several experiments, including spreading pectin on the inner bark of persimmon trees to capture cesium, and spraying leaves or injecting trees with potassium solution to drive out cesium. Whenever we return to the Tokyo campus, we have samples to measure. The other students do not talk when they work, so I let them concentrate by trying to look at the overall picture and do whatever is required to help. I believe that I am getting a chance to learn about radioactive cesium through first-hand experiments and to study how humans can coexist with radiation.

Open Access This chapter is distributed under the terms of the Creative Commons Attribution Noncommercial License, which permits any noncommercial use, distribution, and reproduction in any medium, provided the original author(s) and source are credited. 